Technical note

\title{
Validated analytical methodology for the simultaneous determination of a wide range of pesticides in human blood using GC-MS/MS and LC-ESI/MS/MS and its application in two poisoning cases
}

\author{
Octavio P. Luzardo a,b,*, Maira Almeida-González ${ }^{\text {a,b }}$, Norberto Ruiz-Suárez a , Manuel Zumbado a,b, \\ Luis A. Henríquez-Hernández a , María José Meilán ${ }^{\text {b }}$, María Camacho a , Luis D. Boada a,b \\ a Toxicology Unit, Research Institute of Biomedical and Health Sciences (IUIBS), University of Las Palmas de Gran Canaria, 35016 Las Palmas de Gran Canaria, Spain \\ ${ }^{\mathrm{b}}$ Institute of Legal Medicine of Las Palmas, Canary Islands Government, Paseo Blas Cabrera Felipe, s/n 35016 Las Palmas de Gran Canaria, Spain
}

\section{A R T I C L E I N F O}

\section{Article history:}

Received 18 August 2014

Received in revised form 13 April 2015

Accepted 15 April 2015

\section{Keywords:}

Forensic science

Pesticide poisoning

Forensic toxicology

Multiresidue

GC-MS/MS

LC-MS/MS

\begin{abstract}
A B S T R A C T
Pesticides are frequently responsible for human poisoning and often the information on the involved substance is lacking. The great variety of pesticides that could be responsible for intoxication makes necessary the development of powerful and versatile analytical methodologies, which allows the identification of the unknown toxic substance. Here we developed a methodology for simultaneous identification and quantification in human blood of 109 highly toxic pesticides. The application of this analytical scheme would help in minimizing the cost of this type of chemical identification, maximizing the chances of identifying the pesticide involved. In the methodology that we present here, we use a liquid-liquid extraction, followed by one single purification step, and quantitation of analytes by a combination of liquid and gas chromatography, both coupled to triple quadrupole mass spectrometry, which is operated in the mode of multiple reaction monitoring. The methodology has been fully validated, and its applicability has been demonstrated in two recent cases involving one self-poisoning fatality and one non-fatal homicidal attempt.
\end{abstract}

(c) 2015 The Chartered Society of Forensic Sciences. Published by Elsevier Ireland Ltd. All rights reserved.

\section{Introduction}

The large group of pesticides, which are widely used throughout the world primarily to control pests affecting crops, is often implicated in human poisoning [1]. Morbidity and mortality attributable to these substances vary from country to country, depending on many variables such as the level of socioeconomic development, accessibility to these chemicals, and the importance of the agricultural sector. Fatalities involving pesticides are a consequence of accidents, self-injury or more rarely homicides, and range from less than $1 \%$ of deaths from poisoning in EU countries [1,2] to up to 71\% of all violent deaths in the Western Pacific and Southeast Asia [3-5]. In all these cases chemical analysis to investigate the poison involved is mandatory, and the forensic laboratory is facing a challenge because in most cases there is no information on what the substance involved was. The main difficulties of this type of toxicological identifications are the wide variety of biological matrices that are sent to the laboratory, often in advanced state of decomposition, along with the wide range of pesticides to which the poisoning could be attributed.

\footnotetext{
* Corresponding author at: Toxicology Unit, Dpt. of Clinical Sciences, Health Sciences Faculty, University of Las Palmas de Gran Canaria, Plaza Dr. Pasteur, s/n 35016 Las Palmas de Gran Canaria, Spain. Tel.: + 34928451 424; fax: + 34928451416.

E-mail address: operez@dcc.ulpgc.es (O.P. Luzardo).
}

In recent years the use of chromatographic techniques (HPLC or GC) combined with detection of analytes by mass spectrometry (MS/MS) has been considered as a very useful tool in forensic toxicology laboratories, since it enables high selectivity along with a very low detection limits. Triple quadrupole mass spectrometers (QqQ) allow operating in the mode of selective multiple reaction monitoring (SRM). This allows the monitoring of parent ions fragmenting into product ions. This mode of operation improves selectivity and sensitivity of the determination, in comparison with one-stage mass spectrometry. With this technique, the virtual elimination of isobaric interferences is allowed, as well as a significant decrease in chemical noise from the matrix [6]. The use of any of these analytical techniques is currently seen as a practical way to overcome the difficulties posed by complex biological matrices, which may contain an excessive amount of potentially interfering substances, such as fat, protein, sugars, and chemicals [7]. In addition, high acquisition speed in the MRM mode allows the development of methods for the simultaneous analysis of tens or even hundreds of compounds belonging to different chemical classes [8-12].

It needs to be emphasized that when the information of the pesticide involved is lacking it is generally difficult to conduct thorough analytical investigations in complex biological matrices such as ante- or postmortem blood, and usually several complementary analyses are needed. That is why all the techniques of high sensitivity and specificity that allow the simultaneous analysis of a wide series of chemicals of high 
toxicity may contribute to reducing the costs associated with this type of analytical, as well as to increase the chances of identifying the "unknown toxic substance". In this work we have developed an analytical method for the detection and quantification of 109 pesticides in human blood. The pesticides have been selected on the basis of both, their high toxicity to humans [13], and the frequency with which they are involved in cases of poisoning [14,15]. This methodology is based on a liquid-liquid extraction, clean-up, chromatographic separation, and detection by QqQ operated in the MRM mode, and has been successfully applied to the detection of the toxicant involved in 2 recent cases of poisoning by an unknown pesticide that were submitted to our laboratory. Besides, this methodology has been also applied in our laboratory to the identification of the pesticides in matrices other than blood [16].

\section{Materials and methods}

\subsection{Chemicals and reagents}

Acetone, acetonitrile, cyclohexane, dichloromethane, ethyl acetate, and methanol (>99.9\%) were purchased from Fisher Scientific (Leicestershire, United Kingdom). Ultrapure (UP) water was obtained from a Milli-Q Gradient A10 (Millipore, Molsheim, France). Blank blood was purchased from Medichem (Medidrug $®$ Basis Line, Medichem, Germany). All the pesticide standards (purity from 97\% to 99.5\%), as well as the internal standards (ISs, aldicarb-D3, carbofuran-D3, chlorfenvinphos-D10, chloropropham, chlorpyriphos-D10, diazinonD10, heptachloro epoxide cis, and thiobencarb), were purchased from Dr Ehrenstorfer Reference Materials (Augsburg, Germany). We prepared stock solutions of target compounds $(0.1$ and $1 \mathrm{mg} / \mathrm{mL})$ in cyclohexane or acetonitrile. Stock solutions were stored at $-20^{\circ} \mathrm{C}$. From these stock solutions matrix-matched calibration curves were prepared $(0.5 \mathrm{ng} / \mathrm{mL}$ to $500 \mathrm{ng} / \mathrm{mL}$ ) using blank blood. For the fortification experiments we used mixtures of all the standards in acetone $(10 \mu \mathrm{g} / \mathrm{mL}$ and $500 \mathrm{ng} / \mathrm{mL})$.

\subsection{Pesticide selection}

A wide variety of pesticides belonging to different chemical classes are currently used in agricultural practices but, because of their high toxicity, several restrictions have been applied and most of the most toxic compounds are nowadays banned. Nevertheless, it has been shown that legal and commercial restrictions have not influenced the intentional illegal use of some pesticides as poisons [16,17]. For this reason for the selection of the 109 pesticides included in this multiresidue method we have mainly taken into account their known toxicities for either humans and other mammals (Table 1), and also according to the available data, the frequency with which these compounds have been implicated in human poisonings [14,15], regardless of whether their use is currently allowed or not.

\subsection{Extraction and cleanup procedure}

A liquid-liquid extraction procedure was developed for human whole blood. For the extraction, $2 \mathrm{~mL}$ of the sample $(\mathrm{pH}=7.0)$ was placed in 50-mL polypropylene centrifuge tubes. Next, $5 \mathrm{~mL}$ of ultrapure water and $50 \mu \mathrm{L}$ of the ISs solution at $1 \mu \mathrm{g} / \mathrm{mL}$ were added and thoroughly vortexed. $10 \mathrm{~mL}$ of a mixture of dichloromethane/ ethyl acetate/acetone (50/30/20) were added to the tubes. The tubes were then placed in an orbital shaker for $10 \mathrm{~min}$. The tubes were then sonicated for $5 \mathrm{~min}$. The samples were centrifuged at $5000 \mathrm{rpm}$, $5 \mathrm{~min}, 20^{\circ} \mathrm{C}$, and the supernatant collected. The samples were then placed under a gentle nitrogen stream to evaporate the solvent. The concentrated extracts were re-dissolved in $3 \mathrm{~mL}$ of cyclohexane in Eppendorf tubes.

After the extraction an additional cleanup step by freezing centrifugation was performed to minimize the content of interfering substances (mainly lipids). The tubes were placed in a $-82^{\circ} \mathrm{C}$ freezer for $20 \mathrm{~min}$, and then centrifuged at $5000 \mathrm{rpm}, 5 \mathrm{~min},-10{ }^{\circ} \mathrm{C}$. The frozen lipids remained in the bottom of the tube and thus separated from the pesticides dissolved in the supernatant, which was carefully removed. This procedure was performed three times, and the resulting supernatant was divided into two 1-mL aliquots. One aliquot was directly used for GC-MS/MS. The other was evaporated and re-dissolved in acetonitrile for LC-MS/MS analysis.

\subsection{GC-QqQ-MS/MS analysis}

For the GC-MS-MS detection of the pesticides included in this study we used a Trace GC Ultra tandem coupled with a TSQ XLS triple quadrupole (QqQ) mass spectrometer instrument (Thermo Fisher Scientific Inc., Waltham, MA, USA). As the stationary phase a $30 \mathrm{~m} \times 0.25-\mathrm{mm}$ i.d., $0.25 \mu \mathrm{m}$ film thickness column was used (BPX5, SGE Inc., Austin, TX, USA). Helium (99.999\%) was used as the carrier gas at a constant flow of $1 \mathrm{~mL} / \mathrm{min}$. The 61 -min oven temperature program was: $60{ }^{\circ} \mathrm{C}$ held for $1 \mathrm{~min}$, ramped to $160{ }^{\circ} \mathrm{C}$ at $15{ }^{\circ} \mathrm{C} / \mathrm{min}$, then to $230{ }^{\circ} \mathrm{C}$ at $2.3^{\circ} \mathrm{C} / \mathrm{min}$, and finally to $290{ }^{\circ} \mathrm{C}$ at $5^{\circ} \mathrm{C} / \mathrm{min}$ and held for $8.9 \mathrm{~min}$. The injector temperature was set al $270{ }^{\circ} \mathrm{C}$ and the transfer line was heated to $310{ }^{\circ} \mathrm{C}$. The injection volume was $1 \mu \mathrm{l}$ in the splitless mode.

The GC was tandem-coupled to a TSQ XLS QqQ mass spectrometer, which was used for the detection and quantification of the 90 most apolar pesticides. An electron ionization (EI)-MS/MS library was specially created for the target analytes under our experimental conditions. We calibrated the mass spectrometer scale with perfluorotributylamine on a weekly basis to ensure an optimal response over time and proper mass assignments. The instrument control, data acquisition and data analysis was performed using the Thermo Fisher Xcalibur software (Ver. 2.0.1).

We constructed a timed MRM method for the simultaneous analysis of 90 pesticides plus ISs in a single run. Matrix-matched calibration curves contained all of the target compounds except for the ISs at each level $(0.5$ to $500 \mathrm{ng} / \mathrm{mL}$ ). The operation conditions of the mass spectrometer were: electron impact ionization (70 eV) in MRM; emission current, $50 \mu \mathrm{A}$; ionization source temperature, $220^{\circ} \mathrm{C}$; electron multiplier voltage, $1500 \mathrm{~V}$; scan width, 0.15 ; scan time, $0.05 \mathrm{~s}$; and peak width, $m / z$ 0.7 Da. Argon (99.99\%) was used as the collision gas at $0.2 \mathrm{~Pa}$.

\subsection{LC-MS-MS analysis}

Because some of the most relevant pesticides causing poisoning in humans, such as carbofuran and aldicarb, can only be analyzed by liquid chromatography due to their high polarity (unless prior derivatization is performed), we developed a complementary method by LC-MS-MS. In this second method we included 19 compounds. Some of them can be analyzed both, by liquid chromatography and by gas chromatography (such as metamidofos, dimethoate, or pirimicarb), but we chose the technique with which a higher sensitivity for each one of them is achieved. However, we have avoided duplicating them in both methods to minimize the number of transitions and to gain sensitivity.

For the LC-MS-MS detection, we used an Accela LC tandem coupled to a TSQ Quantum Max QqQ mass spectrometer instrument equipped with an H-ESI II electrospray ionization source (Thermo Fisher Scientific Inc.). As the stationary phase we used an analytic Synergi Hydro-RP column ( $4.0 \mu \mathrm{m}, 150 \times 4.6 \mathrm{~mm}$; Phenomenex, Torrance, CA, USA). We used the following mobile phases for LC: (A) $7.5 \mathrm{mM}$ ammonium formate in ultrapure water; (B) methanol (HPLC-MS grade); and (C) $2 \%$ formic acid. The solvent flow was $1000 \mu \mathrm{l} / \mathrm{min}$. The injection volume was $25 \mu \mathrm{l}$. During the entire run (26 min), solvent $C$ was set at $2.5 \%$. The infusion of the other two mobile phases was programmed as a gradient as follows: $0-12 \mathrm{~min}: 87.5 \% \mathrm{~A} \rightarrow 7.5 \% \mathrm{~A} ; 12-16 \mathrm{~min}$ : 7.5\% A; $16.0-16.2$ min: 7.5\% A $\rightarrow$ 87.5\% A; and 16.2-25.0 min: 87.5\% A. 
Table 1

Acute toxicity values $(\mathrm{mg} / \mathrm{kg})$ and toxic and lethal blood concentrations $(\mu \mathrm{g} / \mathrm{mL})$ of the pesticides included in the methodology.

\begin{tabular}{|c|c|c|c|c|c|c|c|c|c|c|c|}
\hline \multirow[t]{2}{*}{ Compound } & \multicolumn{3}{|c|}{ Toxicity } & \multirow[t]{2}{*}{ Compound } & \multicolumn{3}{|c|}{ Toxicity } & \multirow[t]{2}{*}{ Compound } & \multicolumn{3}{|l|}{ Toxicity } \\
\hline & $\mathrm{LD}_{50}{ }^{\mathrm{a}}$ & [Toxic $^{\mathrm{b}}$ & $\overline{\text { [Lethal] }^{\mathrm{b}}}$ & & $\mathrm{LD}_{50}$ & {$[\text { Toxic }]^{b}$} & $\overline{\text { [Lethal] }^{\mathrm{b}}}$ & & $\mathrm{LD}_{50}$ & 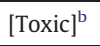 & [Lethal] $^{\mathrm{b}}$ \\
\hline Acephate & 321.0 & $>200$ & - & Dieldrin & 65 & 0.15 & 0.5 & Mevinphos & 4.0 & - & - \\
\hline Aldicarb & 1.9 & 1.5 & 6.1 & Dimefox & 3.5 & - & - & Monocrotophos & 15.0 & - & 12 \\
\hline Aldrin & 65.0 & 0.005 & 0.7 & Dimethoate & 220.0 & - & 4 & Nuarimol & 2450.0 & - & - \\
\hline Allethrin & 370.0 & - & - & Dioxathion & 10.0 & 0.2 & - & Omethoate & 50.0 & - & 3.2 \\
\hline Amitraz & 100.0 & 0.6 & - & Disulfoton & 5.0 & - & - & Oxamyl & 30.0 & 0.23 & - \\
\hline Azinphos ethyl & 12.0 & - & 0.9 & Ediphenphos & 100.0 & - & 1.4 & Parathion ethyl & 0.9 & - & 0.5 \\
\hline Azinphos methyl & 10.0 & - & - & Endosulfan sulfate & 18.0 & - & - & Parathion methyl & 57.0 & - & - \\
\hline Bendiocarb & 35.0 & 1 & 40 & Endosulfan, alpha & 26.0 & 0.5 & 2.8 & Phenthoate & 138.0 & - & - \\
\hline Benfuracarb & 102.0 & - & - & Endosulfan, beta & 26.0 & 0.5 & 2.8 & Phorate & 20.0 & - & 0.83 \\
\hline Bifenthrin & 54.5 & - & - & Endrin & 3.0 & 0.01 & - & Phosalone & 112.0 & - & - \\
\hline Bromophos ethyl & 125.0 & - & 1.6 & EPN & 20.0 & - & 0.8 & Phosmet & 40.0 & - & - \\
\hline Bromoxynil & 78.0 & 20 & - & Ethion & 13.0 & - & - & Phosphamidon & 6.0 & - & - \\
\hline Cadusafos & 71.4 & 5 & 6 & Etoprophos & 34.0 & - & - & Phoxim & 250 & - & - \\
\hline Carbaryl & 150.0 & - & 2 & Famphur & 59.0 & - & - & Pirimicarb & 100.0 & 3.7 & 32.8 \\
\hline Carbofuran & 10.2 & 0.06 & 0.4 & Fenamiphos & 10.0 & - & - & Pirimiphos ethyl & 25.0 & - & - \\
\hline Carbophenothion & 14.0 & - & - & Fenitrothion & 142.0 & 1 & - & Pirimiphos methyl & 1150.0 & - & - \\
\hline Carbosulfan & 115.0 & - & - & Fensulfothion & 2.2 & - & - & Profenofos & 116.0 & - & 1.2 \\
\hline Carboxin & 430.0 & - & - & Fenthion & 46.2 & 1 & - & Propachlor & 392.0 & - & - \\
\hline Chlordane, cis & 50.0 & 0.005 & 2 & Flucythrinate & 76.0 & - & - & Propaphos & 61 & - & - \\
\hline Chlordane, trans & 50.0 & 0.005 & 2 & Fonofos & 3.0 & - & - & Propetamphos & 130.0 & - & - \\
\hline Chlorfenvinphos & 20.0 & - & 0.1 & Formothion & 175.0 & - & - & Propoxur & 51.2 & 0.12 & - \\
\hline Chlormephos & 12.5 & - & - & Heptachlor & 50.0 & - & - & Pyrazophos & 184.0 & - & - \\
\hline Chlorpyrifos & 60.0 & 0.2 & 1.6 & Heptenophos & 117.0 & - & - & Quinalphos & 75.0 & - & 4.5 \\
\hline Chlorpyriphos methyl & 2000.0 & - & - & Imidacloprid & 98.0 & - & 2.1 & Resmethrin & 250.0 & - & - \\
\hline Chlorthiophos & 20.0 & - & - & Isazophos & 27.0 & - & - & Sulfotep & 22.0 & 0.08 & - \\
\hline Cifluthrin & 300.0 & 3.1 & 37.4 & Isobenzan & 5.0 & 0.03 & - & Sulprofos & 70.0 & - & - \\
\hline Cyanazine & 141.0 & - & - & Isofenphos & 91.5 & - & - & Tebufenpyrad & 210.0 & - & - \\
\hline Cyanophos & 215.0 & - & - & Isoxathion & 112.0 & - & - & Tefluthrin & 22.0 & 0.9 & 7.1 \\
\hline Cyproconazole & 352.0 & - & - & Leptophos & 65.0 & - & - & TEPP & 2.3 & - & 2.7 \\
\hline Dazomet & 415.0 & - & - & Lindane & 25.0 & 0.3 & 1.3 & Terbufos & 3.5 & - & - \\
\hline DDT & 200.0 & 1 & - & Malathion & 53.0 & 0.5 & 1 & Tetrachlorvinphos & 420.0 & - & - \\
\hline Deltamethrin & 22.0 & 0.2 & 9.3 & Mephospholan & 11.0 & - & - & Thiometon & 37.0 & - & - \\
\hline Diallate & 395.0 & - & - & Metamidofos & 18.5 & - & 13.5 & Thionazin & 5.0 & - & 0.8 \\
\hline Diazinon & 76.0 & 0.05 & 0.97 & Methidathion & 25.0 & 0.1 & - & Triazophos & 57.0 & - & - \\
\hline Dichlone & 160.0 & - & - & Methiocarb & 16.0 & - & - & Trichloronat & 10.0 & 0.15 & - \\
\hline Dichlorphos & 61.0 & - & 29 & Metolcarb & 109.0 & - & - & & & & \\
\hline Dicrotophos & 11.0 & 0.3 & 2.8 & Methomyl & 24.9 & - & 0.45 & & & & \\
\hline
\end{tabular}

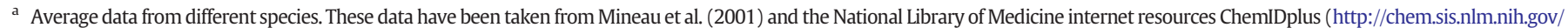
chemidplus/chemidheavy.jsp) and Hazardous Substances Data Bank (http://toxnet.nlm.nih.gov/cgi-bin/sis/htmlgen?HSDB).

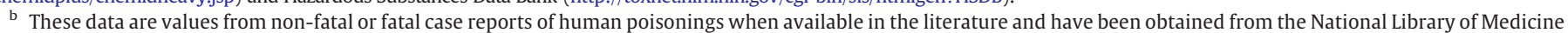

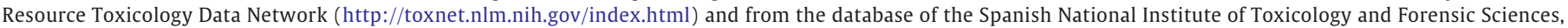
available in http://busca-tox.com/.

The LC was tandem-coupled to a TSQ Quantum Max QqQ mass spectrometer, which was used for the detection and quantification of the 19 pesticides. As the ionization source we used a heated electrospray H-ESI II (Thermo Fisher Scientific Inc., Waltham, USA). The mass spectrometer was programmed according the following parameters: skimmer offset $(10 \mathrm{~V})$, sheath gas pressure (15 arbitrary units, a.u.), capillary temperature $\left(250{ }^{\circ} \mathrm{C}\right)$, spray voltage $(3000 \mathrm{~V})$, and vaporization temperature $\left(180^{\circ} \mathrm{C}\right)$. The spectrometer was programed in the positive ionization mode. Argon (99.99\%) was used as the collision gas at $0.25 \mathrm{~Pa}$.

The MRM method was constructed for these pesticides plus ISs by means of the infusion of pure standard solutions in methanol into the ionization source. A matrix-matched calibration curve was constructed for all compounds (except for the ISs) from 0.5 to $500 \mathrm{ng} / \mathrm{mL}$.

\subsection{Validation}

Blank whole blood (Medidrug Basis-Line VB, Medichem, Germany) was used for the validation experiments. For the fortification experiments $40 \mu \mathrm{L}$ of a $10 \mu \mathrm{g} / \mathrm{L}$ or $500 \mathrm{ng} / \mathrm{L}$ of a mixture of all of the pesticides in acetone were added to $2 \mathrm{~mL}$ of whole blood to obtain concentrations of $200 \mu \mathrm{g} / \mathrm{kg}$ and $10 \mu \mathrm{g} / \mathrm{kg}$, respectively. The samples were thoroughly mixed and allowed to stand at room temperature for $4 \mathrm{~h}$ to ensure that the analytes were homogenously distributed throughout the sample. The matrix effect was determined in quintuplicate by comparing the obtained concentrations between the spiked blood and the same concentrations of the pesticides prepared in the dissolvent. The recovery was calculated as the quotient of signals obtained from the spiked samples and the matrix matched standards. The intra- and inter-day precisions (five successive days) were also determined from the same experiments.

The analyte concentration that produced a peak signal of ten times the background noise from the chromatogram was set as the method limit of quantification (LOQ). Quantifications were based on the peak areas. Calibration curves were constructed using a least-squares linear regression from the injection of samples spiked with solutions to give final concentrations ranging from 0.5 to $500 \mu \mathrm{g} / \mathrm{kg}$

\subsection{Quality control}

All the measurements were performed in triplicate, and the values used for calculations were the mean of the three values. In each batch of samples, two controls were included every 12 samples: a reagent blank consisting of a vial containing only cyclohexane and an internal laboratory quality control (QC) consisting of blank whole blood spiked with a mixture of all of the pesticides $(20 \mu \mathrm{g} / \mathrm{kg})$, and processed with the same method as the samples. The results were considered acceptable when the quantification of the analytes in the QC was within $15 \%$ of the deviation of the theoretical value. 
Table 2

Method settings and validation parameters $(n=5)$ obtained for the 109 pesticides at the concentration of $0.2 \mu \mathrm{g} / \mathrm{mL}$ in human blood.

\begin{tabular}{|c|c|c|c|c|c|c|c|c|c|c|}
\hline \multirow[t]{2}{*}{$\mathrm{N}^{\circ}$} & \multirow[t]{2}{*}{ Compound } & \multicolumn{6}{|c|}{ Mass spectrometry settings } & \multicolumn{3}{|c|}{ Validation parameters } \\
\hline & & RT & $\begin{array}{l}\text { First transition } \\
m / z \rightarrow m / z\end{array}$ & $\begin{array}{l}\mathrm{CE} \\
(\mathrm{V})\end{array}$ & $\begin{array}{l}\text { Second transition } \\
m / z \rightarrow m / z\end{array}$ & $\begin{array}{l}\mathrm{CE} \\
(\mathrm{V})\end{array}$ & IPs & $\begin{array}{l}\text { LOQ } \\
(\mu \mathrm{g} / \mathrm{mL})\end{array}$ & $\begin{array}{l}\text { Linearity } \\
\left(\mathrm{R}^{2}\right)\end{array}$ & $\begin{array}{l}\text { Average recovery } \\
\left(\mathrm{RSD}^{\mathrm{a}, \mathrm{b}}\right)(\%)\end{array}$ \\
\hline \multicolumn{11}{|c|}{ LC-MS/MS method } \\
\hline 1 & Metamidofos & 2.26 & $142.1 \rightarrow 94.0$ & 14 & $142.1 \rightarrow 125.0$ & 16 & 4 & 0.01 & 0.9985 & $78(14,11)$ \\
\hline 2 & Oxamyl & 2.78 & $237.2 \rightarrow 163.0$ & 14 & $237.2 \rightarrow 196.0$ & 18 & 4 & 0.03 & 0.9824 & $93(8,10)$ \\
\hline 3 & Phoxim & 4.03 & $300.1 \rightarrow 129.3$ & 18 & $300.1 \rightarrow 283.0$ & 10 & 4 & 0.01 & 0.9959 & $97(6,9)$ \\
\hline 4 & Acephate & 4.51 & $184.1 \rightarrow 125.0$ & 16 & $184.1 \rightarrow 143.0$ & 5 & 4 & 0.03 & 0.9816 & $95(7,11)$ \\
\hline 5 & Omethoate & 5.03 & $214.0 \rightarrow 155.0$ & 19 & $214.0 \rightarrow 183.0$ & 13 & 4 & 0.05 & 0.9904 & $96(11,14)$ \\
\hline 6 & Methomyl & 6.71 & $163.1 \rightarrow 88.1$ & 11 & $163.1 \rightarrow 106.0$ & 12 & 4 & 0.02 & 0.9934 & $97(8,12)$ \\
\hline 7 & Imidacloprid & 8.05 & $256.1 \rightarrow 175.0$ & 18 & $256.1 \rightarrow 209.0$ & 16 & 4 & 0.01 & 0.9813 & $88(8,10)$ \\
\hline 8 & Dimethoate & 8.64 & $230.0 \rightarrow 125.0$ & 23 & $230.0 \rightarrow 199.0$ & 11 & 4 & 0.05 & 0.9890 & $92(8,7)$ \\
\hline 9 & Aldicarb & 9.38 & $208.0 \rightarrow 89.2$ & 19 & $208.0 \rightarrow 116.2$ & 10 & 4 & 0.01 & 0.9906 & $94(9,11)$ \\
\hline 10 & Carbofuran & 10.34 & $222.0 \rightarrow 123.1$ & 25 & $222.0 \rightarrow 137.5$ & 24 & 4 & 0.01 & 0.9899 & $97(4,7)$ \\
\hline 11 & Propoxur & 10.76 & $210.0 \rightarrow 111.2$ & 18 & $210.0 \rightarrow 168.0$ & 11 & 4 & 0.01 & 0.9972 & $101(9,13)$ \\
\hline 12 & Carbaryl & 11.20 & $202.1 \rightarrow 127.0$ & 33 & $202.1 \rightarrow 145.1$ & 13 & 4 & 0.01 & 0.9948 & $82(4,8)$ \\
\hline 13 & Pirimicarb & 11.33 & $239.1 \rightarrow 72.3$ & 27 & $239.1 \rightarrow 182.1$ & 16 & 4 & 0.03 & 0.9982 & $96(8,11)$ \\
\hline 14 & Carboxin & 11.49 & $236.1 \rightarrow 93.2$ & 33 & $236.1 \rightarrow 143.0$ & 15 & 4 & 0.03 & 0.9929 & $71(11,9)$ \\
\hline 15 & Bromoxynil & 12.01 & $275.9 \rightarrow 79.2$ & 29 & $275.9 \rightarrow 81.1$ & 33 & 4 & 0.05 & 0.9994 & $92(9,11)$ \\
\hline 16 & Methiocarb & 12.97 & $226.0 \rightarrow 121.0$ & 19 & $226.0 \rightarrow 169.0$ & 8 & 4 & 0.05 & 0.9856 & $97(12,15)$ \\
\hline 17 & Cyproconazole & 13.46 & $292.1 \rightarrow 70.3$ & 17 & $292.1 \rightarrow 125.1$ & 34 & 4 & 0.01 & 0.9908 & $99(11,9)$ \\
\hline 18 & Benfuracarb & 15.02 & $411.2 \rightarrow 190.1$ & 13 & $411.2 \rightarrow 252.3$ & 15 & 4 & 0.05 & 0.9873 & $85(5,8)$ \\
\hline 19 & Profenofos & 15.52 & $373.0 \rightarrow 302.8$ & 18 & $373.0 \rightarrow 344.8$ & 13 & 4 & 0.03 & 0.9814 & $93(14,11)$ \\
\hline \multicolumn{11}{|c|}{ GC-MS/MS method } \\
\hline 20 & Dimefox & 5.33 & $154.1 \rightarrow 58.0$ & 10 & $154.1 \rightarrow 111.1$ & 10 & 4 & 0.01 & 0.9834 & $91(11,9)$ \\
\hline 21 & Dichlorphos & 7.61 & $185.0 \rightarrow 109.0$ & 15 & $185.0 \rightarrow 127.0$ & 12 & 4 & 0.01 & 0.9921 & $90(14,12)$ \\
\hline 22 & Mevinphos & 9.72 & $192.0 \rightarrow 127.0$ & 12 & $192.0 \rightarrow 164.0$ & 10 & 4 & 0.01 & 0.9953 & $101(10,14)$ \\
\hline 23 & Chlormephos & 9.94 & $154.0 \rightarrow 121.0$ & 5 & $154.0 \rightarrow 121.0$ & 14 & 4 & 0.02 & 0.9878 & $105(9,12)$ \\
\hline 24 & Metolcarb & 10.56 & $108.1 \rightarrow 79.0$ & 10 & $108.1 \rightarrow 107.1$ & 10 & 4 & 0.02 & 0.9877 & $103(14,11)$ \\
\hline 25 & Heptenophos & 12.26 & $250.0 \rightarrow 124.0$ & 10 & $250.0 \rightarrow 215.0$ & 4 & 4 & 0.01 & 0.9887 & $93(6,8)$ \\
\hline 26 & Thionazin & 12.96 & $192.0 \rightarrow 96.0$ & 10 & $248.0 \rightarrow 140.0$ & 10 & 5 & 0.05 & 0.9964 & $100(8,9)$ \\
\hline 27 & TEPP & 13.07 & $263.1 \rightarrow 179.1$ & 15 & $263.1 \rightarrow 235.1$ & 5 & 4 & 0.01 & 0.9987 & $94(12,10)$ \\
\hline 28 & Propachlor & 13.25 & $176.1 \rightarrow 120.0$ & 10 & $196.1 \rightarrow 120.0$ & 10 & 5 & 0.02 & 0.9913 & $96(8,11)$ \\
\hline 29 & Etoprophos & 13.70 & $158.0 \rightarrow 114.0$ & 10 & $158.0 \rightarrow 130.0$ & 10 & 4 & 0.01 & 0.9978 & $95(6,8)$ \\
\hline 30 & Sulfotep & 14.50 & $322.0 \rightarrow 202.0$ & 15 & $322.0 \rightarrow 294.0$ & 10 & 4 & 0.01 & 0.9889 & $92(11,14)$ \\
\hline 31 & Dicrotophos & 14.61 & $127.0 \rightarrow 95.0$ & 10 & $127.0 \rightarrow 109.0$ & 10 & 4 & 0.01 & 0.9887 & $101(9,12)$ \\
\hline 32 & Bendiocarb & 14.79 & $166.1 \rightarrow 151.1$ & 15 & $223.1 \rightarrow 166.1$ & 15 & 5 & 0.05 & 0.9856 & $93(7,9)$ \\
\hline 33 & Cadusafos & 14.93 & $159.1 \rightarrow 97.0$ & 20 & $159.1 \rightarrow 131.0$ & 10 & 4 & 0.01 & 0.9948 & $941(8,12)$ \\
\hline 34 & Phorate & 15.24 & $260.0 \rightarrow 75.0$ & 5 & $260.0 \rightarrow 231.0$ & 8 & 4 & 0.01 & 0.9932 & $93(6,8)$ \\
\hline 35 & Diallate & 15.30 & $236.0 \rightarrow 152.0$ & 20 & $236.0 \rightarrow 194.0$ & 15 & 4 & 0.02 & 0.9921 & $95(10,11)$ \\
\hline 36 & Monocrotophos & 15.80 & $127.0 \rightarrow 95.0$ & 20 & $127.0 \rightarrow 109.3$ & 25 & 4 & 0.01 & 0.9904 & $92(9,11)$ \\
\hline 37 & Thiometon & 15.85 & $88.0 \rightarrow 60.0$ & 15 & $248.0 \rightarrow 88.0$ & 15 & 5 & 0.01 & 0.9878 & $87(7,7)$ \\
\hline 38 & Dazomet & 16.56 & $89.0 \rightarrow 75.0$ & 20 & $162.0 \rightarrow 89.0$ & 8 & 5 & 0.05 & 0.9877 & $89(8,11)$ \\
\hline 39 & Dioxathion & 17.13 & $125.0 \rightarrow 97.0$ & 15 & $197.0 \rightarrow 141.0$ & 15 & 5 & 0.01 & 0.9887 & $101(7,9)$ \\
\hline 40 & Lindane & 17.16 & $216.9 \rightarrow 180.9$ & 15 & $218.9 \rightarrow 182.9$ & 15 & 5 & 0.005 & 0.9964 & $92(11,9)$ \\
\hline 41 & Propetamphos & 17.35 & $236.1 \rightarrow 166.1$ & 15 & $236.1 \rightarrow 194.1$ & 5 & 5 & 0.01 & 0.9987 & $94(14,12)$ \\
\hline 42 & Terbufos & 17.37 & $231.0 \rightarrow 175.0$ & 15 & $231.0 \rightarrow 203.0$ & 10 & 4 & 0.01 & 0.9945 & $101(10,14)$ \\
\hline 43 & Chlorfenvinfos & 17.60 & $267.0 \rightarrow 159.0$ & 15 & $323.0 \rightarrow 269.0$ & 10 & 5 & 0.01 & 0.9995 & $103(14,11)$ \\
\hline 44 & Cyanofos & 17.60 & $243.0 \rightarrow 109.0$ & 12 & $243.0 \rightarrow 127.0$ & 15 & 4 & 0.01 & 0.9883 & $94(6,11)$ \\
\hline 45 & Fonofos & 17.69 & $137.0 \rightarrow 109.0$ & 10 & $246.0 \rightarrow 137.0$ & 10 & 5 & 0.01 & 0.9978 & $92(4,9)$ \\
\hline 46 & Diazinon & 17.81 & $179.1 \rightarrow 127.0$ & 15 & $179.1 \rightarrow 137.1$ & 15 & 4 & 0.01 & 0.9907 & $92(9,6)$ \\
\hline 47 & Disulfoton & 18.32 & $274.0 \rightarrow 88.0$ & 10 & $274.0 \rightarrow 245.0$ & 10 & 4 & 0.01 & 0.9889 & $94(6,11)$ \\
\hline 48 & Tefluthrin & 18.40 & $197.0 \rightarrow 141.0$ & 15 & $197.0 \rightarrow 161.0$ & 10 & 4 & 0.02 & 0.9887 & $91(7,5)$ \\
\hline 49 & Isazophos & 18.40 & $257.0 \rightarrow 119.0$ & 15 & $257.0 \rightarrow 162.0$ & 15 & 4 & 0.01 & 0.9856 & $84(4,6)$ \\
\hline 50 & Dichlone & 18.58 & $191.0 \rightarrow 135.0$ & 15 & $226.0 \rightarrow 191.0$ & 10 & 5 & 0.02 & 0.9819 & $89(8,7)$ \\
\hline 51 & Formothion & 19.94 & $224.0 \rightarrow 125.0$ & 15 & $224.0 \rightarrow 196.0$ & 10 & 4 & 0.01 & 0.9881 & $94(5,9)$ \\
\hline 52 & Phosphamidon & 20.08 & $264.0 \rightarrow 127.0$ & 15 & $264.0 \rightarrow 127.0$ & 15 & 4 & 0.01 & 0.9883 & $91(6,10)$ \\
\hline 53 & Chlorpyrifos methyl & 20.57 & $285.9 \rightarrow 93.0$ & 25 & $285.9 \rightarrow 272.9$ & 13 & 4 & 0.01 & 0.9890 & $88(8,11)$ \\
\hline 54 & Parathion methyl & 21.10 & $263.0 \rightarrow 109.0$ & 15 & $263.0 \rightarrow 127.0$ & 15 & 4 & 0.01 & 0.9992 & $89(6,8)$ \\
\hline 55 & Heptachlor & 21.36 & $338.8 \rightarrow 267.9$ & 15 & $338.8 \rightarrow 303.8$ & 15 & 4 & 0.005 & 0.9899 & $94(8,6)$ \\
\hline 56 & Fenitrothion & 22.74 & $277.0 \rightarrow 109.0$ & 20 & $277.0 \rightarrow 260.0$ & 15 & 4 & 0.01 & 0.9972 & $90(15,12)$ \\
\hline 57 & Pirimifos methyl & 23.19 & $290.1 \rightarrow 125.0$ & 15 & $290.1 \rightarrow 233.1$ & 10 & 4 & 0.01 & 0.9948 & $93(12,8)$ \\
\hline 58 & Malathion & 23.27 & $173.0 \rightarrow 127.0$ & 10 & $173.0 \rightarrow 145.0$ & 5 & 4 & 0.01 & 0.9982 & $94(12,14)$ \\
\hline 59 & Chlorpyrifos & 23.60 & $197.0 \rightarrow 169.0$ & 15 & $199.0 \rightarrow 171.0$ & 15 & 5 & 0.01 & 0.9929 & $92(11,13)$ \\
\hline 60 & Aldrin & 23.60 & $262.9 \rightarrow 192.9$ & 32 & $262.9 \rightarrow 227.9$ & 26 & 4 & 0.003 & 0.9994 & $78(12,8)$ \\
\hline 61 & Fenthion & 24.08 & $278.0 \rightarrow 169.0$ & 20 & $278.0 \rightarrow 245.0$ & 15 & 4 & 0.01 & 0.9856 & $81(7,11)$ \\
\hline 62 & Parathion ethyl & 24.26 & $291.0 \rightarrow 109.0$ & 15 & $291.0 \rightarrow 263.0$ & 10 & 4 & 0.01 & 0.9908 & $87(9,13)$ \\
\hline 63 & Isobenzan & 24.41 & $310.8 \rightarrow 274.8$ & 10 & $312.8 \rightarrow 276.8$ & 10 & 5 & 0.02 & 0.9873 & $82(11,13)$ \\
\hline 64 & Cyanazine & 24.59 & $225.1 \rightarrow 189.1$ & 10 & $225.1 \rightarrow 198.1$ & 10 & 4 & 0.05 & 0.9948 & $75(9,12)$ \\
\hline 65 & Trichloronat & 24.70 & $296.9 \rightarrow 268.9$ & 15 & $299.9 \rightarrow 271.9$ & 15 & 5 & 0.04 & 0.9932 & $93(6,8)$ \\
\hline 66 & Pirimifos ethyl & 26.08 & $333.1 \rightarrow 288.1$ & 20 & $333.1 \rightarrow 318.1$ & 15 & 4 & 0.01 & 0.9921 & $95(8,9)$ \\
\hline 67 & Isofenphos & 26.44 & $255.1 \rightarrow 185.1$ & 10 & $255.1 \rightarrow 213.1$ & 10 & 4 & 0.01 & 0.9904 & $94(12,10)$ \\
\hline 68 & Allethrin & 26.93 & $123.1 \rightarrow 81.1$ & 10 & $136.1 \rightarrow 93.1$ & 10 & 4 & 0.02 & 0.9987 & $92(11,14)$ \\
\hline 69 & Phenthoate & 27.10 & $274.0 \rightarrow 125.0$ & 7 & $274.0 \rightarrow 246.0$ & 10 & 4 & 0.05 & 0.9945 & $91(8,12)$ \\
\hline 70 & Quinalphos & 27.17 & $146.0 \rightarrow 91.0$ & 15 & $146.0 \rightarrow 118.0$ & 15 & 4 & 0.03 & 0.9976 & $90(8,10)$ \\
\hline
\end{tabular}


Table 2 (continued)

\begin{tabular}{|c|c|c|c|c|c|c|c|c|c|c|}
\hline \multirow[t]{2}{*}{$\mathrm{N}^{\circ}$} & \multirow[t]{2}{*}{ Compound } & \multicolumn{6}{|c|}{ Mass spectrometry settings } & \multicolumn{3}{|c|}{ Validation parameters } \\
\hline & & RT & $\begin{array}{l}\text { First transition } \\
m / z \rightarrow m / z\end{array}$ & $\begin{array}{l}\mathrm{CE} \\
(\mathrm{V})\end{array}$ & $\begin{array}{l}\text { Second transition } \\
m / z \rightarrow m / z\end{array}$ & $\begin{array}{l}\mathrm{CE} \\
(\mathrm{V})\end{array}$ & IPs & $\begin{array}{l}\text { LOQ } \\
(\mu \mathrm{g} / \mathrm{mL})\end{array}$ & $\begin{array}{l}\text { Linearity } \\
\left(\mathrm{R}^{2}\right)\end{array}$ & $\begin{array}{l}\text { Average recovery } \\
\left(\mathrm{RSD}^{\mathrm{a}, \mathrm{b}}\right)(\%)\end{array}$ \\
\hline \multicolumn{11}{|c|}{ GC-MS/MS method } \\
\hline 71 & Mephospholan & 27.60 & $196.0 \rightarrow 140.0$ & 15 & $196.0 \rightarrow 168.0$ & 10 & 4 & 0.03 & 0.9995 & $94(8,7)$ \\
\hline 72 & Chlordane, trans & 28.04 & $372.8 \rightarrow 265.9$ & 15 & $374.8 \rightarrow 267.9$ & 16 & 5 & 0.005 & 0.9878 & $93(9,11)$ \\
\hline 73 & Bromophos ethyl & 28.07 & $358.9 \rightarrow 302.9$ & 20 & $358.9 \rightarrow 330.9$ & 10 & 4 & 0.01 & 0.9877 & $92(4,7)$ \\
\hline 74 & Methidathion & 28.23 & $145.0 \rightarrow 58.0$ & 15 & $145.0 \rightarrow 85.0$ & 10 & 4 & 0.01 & 0.9887 & $92(9,13)$ \\
\hline 75 & Propafos & 28.58 & $220.1 \rightarrow 140.0$ & 15 & $304.1 \rightarrow 220.1$ & 15 & 5 & 0.01 & 0.9964 & $77(4,8)$ \\
\hline 76 & Tetrachlorvinphos & 28.64 & $330.9 \rightarrow 109.0$ & 22 & $330.9 \rightarrow 316.0$ & 22 & 4 & 0.01 & 0.9819 & $94(10,8)$ \\
\hline 77 & Endosulfan, alpha & 28.88 & $195.9 \rightarrow 158.9$ & 16 & $195.9 \rightarrow 159.9$ & 15 & 4 & 0.01 & 0.9881 & $91(12,8)$ \\
\hline 78 & Chlordane, cis & 28.90 & $372.8 \rightarrow 265.9$ & 18 & $409.8 \rightarrow 374.8$ & 5 & 5 & 0.005 & 0.9883 & $94(5,9)$ \\
\hline 79 & Fenamiphos & 29.98 & $303.1 \rightarrow 260.1$ & 15 & $303.1 \rightarrow 288.1$ & 15 & 4 & 0.01 & 0.9883 & $94(11,12)$ \\
\hline 80 & Dieldrin & 30.87 & $276.9 \rightarrow 206.9$ & 20 & $276.9 \rightarrow 240.9$ & 10 & 4 & 0.001 & 0.9978 & $92(4,7)$ \\
\hline 81 & Endrin & 32.42 & $262.9 \rightarrow 190.9$ & 25 & $262.9 \rightarrow 192.9$ & 26 & 4 & 0.001 & 0.9889 & $93(9,6)$ \\
\hline 82 & Isoxathion & 32.47 & $177.0 \rightarrow 130.0$ & 15 & $313.0 \rightarrow 177.0$ & 15 & 5 & 0.01 & 0.9887 & $101(6,9)$ \\
\hline 83 & Endosulfan, beta & 33.50 & $195.9 \rightarrow 158.9$ & 16 & $195.9 \rightarrow 159.9$ & 15 & 4 & 0.01 & 0.9856 & $89(11,8)$ \\
\hline 84 & Fensulfothion & 33.84 & $293.0 \rightarrow 97.0$ & 16 & $293.0 \rightarrow 125.0$ & 0 & 4 & 0.01 & 0.9985 & $93(4,11)$ \\
\hline 85 & Ethion & 33.96 & $231.0 \rightarrow 175.0$ & 15 & $231.0 \rightarrow 203.0$ & 15 & 4 & 0.01 & 0.9824 & $91(14,10)$ \\
\hline 86 & Chlorthiophos & 34.26 & $325.0 \rightarrow 269.0$ & 15 & $325.0 \rightarrow 297.0$ & 10 & 4 & 0.01 & 0.9959 & $101(4,8)$ \\
\hline 87 & Sulprofos & 35.31 & $322.0 \rightarrow 139.0$ & 15 & $322.0 \rightarrow 156.0$ & 15 & 4 & 0.01 & 0.9816 & $93(5,7)$ \\
\hline 88 & Triazofos & 35.55 & $161.0 \rightarrow 105.0$ & 13 & $161.0 \rightarrow 134.0$ & 10 & 4 & 0.01 & 0.9904 & $94(9,13)$ \\
\hline 89 & Famphur & 35.87 & $218.0 \rightarrow 109.0$ & 10 & $218.0 \rightarrow 127.0$ & 10 & 4 & 0.01 & 0.9934 & $97(6,9)$ \\
\hline 90 & Carbophenothion & 36.02 & $342.0 \rightarrow 157.0$ & 10 & $342.0 \rightarrow 296.0$ & 5 & 4 & 0.01 & 0.9813 & $89(9,6)$ \\
\hline 91 & Ediphenphos & 36.23 & $173.0 \rightarrow 109.0$ & 15 & $310.0 \rightarrow 173.0$ & 10 & 4 & 0.01 & 0.9890 & $88(12,9)$ \\
\hline 92 & Endosulfan sulfate & 36.38 & $273.9 \rightarrow 236.9$ & 10 & $273.9 \rightarrow 239.0$ & 15 & 4 & 0.01 & 0.9992 & $92(6,9)$ \\
\hline 93 & DDT & 36.77 & $234.9 \rightarrow 165.0$ & 20 & $234.9 \rightarrow 198.9$ & 15 & 4 & 0.002 & 0.9899 & $91(7,11)$ \\
\hline 94 & Nuarimol & 37.69 & $235.1 \rightarrow 139.0$ & 15 & $314.1 \rightarrow 139.0$ & 15 & 5 & 0.05 & 0.9803 & $90(4,6)$ \\
\hline 95 & Resmethrin & 39.00 & $171.1 \rightarrow 128.0$ & 9 & $171.1 \rightarrow 143.0$ & 9 & 4 & 0.05 & 0.9815 & $93(5,9)$ \\
\hline 96 & Carbosulfan & 39.80 & $163.1 \rightarrow 107.1$ & 15 & $163.1 \rightarrow 135.1$ & 10 & 4 & 0.01 & 0.9907 & $87(10,7)$ \\
\hline 97 & Phosmet & 40.66 & $160.0 \rightarrow 104.0$ & 20 & $160.0 \rightarrow 133.0$ & 15 & 4 & 0.01 & 0.9928 & $89(4,8)$ \\
\hline 98 & EPN & 40.75 & $169.0 \rightarrow 77.0$ & 16 & $169.0 \rightarrow 141.0$ & 10 & 4 & 0.005 & 0.9994 & $86(6,9)$ \\
\hline 99 & Bifenthrin & 40.81 & $181.0 \rightarrow 153.0$ & 6 & $181.0 \rightarrow 166.0$ & 15 & 4 & 0.04 & 0.9856 & $91(11,11)$ \\
\hline 100 & Tebufenpyrad & 41.87 & $333.1 \rightarrow 171.1$ & 20 & $333.1 \rightarrow 276.1$ & 10 & 4 & 0.04 & 0.9908 & $94(7,10)$ \\
\hline 101 & Leptophos & 42.96 & $374.9 \rightarrow 359.9$ & 26 & $376.9 \rightarrow 361.9$ & 26 & 5 & 0.01 & 0.9873 & $101(3,7)$ \\
\hline 102 & Phosalone & 43.10 & $182.0 \rightarrow 111.0$ & 15 & $182.0 \rightarrow 138.0$ & 10 & 4 & 0.02 & 0.9972 & $94(8,9)$ \\
\hline 103 & Azinphos methyl & 43.57 & $132.0 \rightarrow 77.0$ & 15 & $160.0 \rightarrow 104.0$ & 10 & 5 & 0.01 & 0.9948 & $91(5,9)$ \\
\hline 104 & Amitraz & 44.30 & $293.2 \rightarrow 147.1$ & 15 & $293.2 \rightarrow 162.1$ & 10 & 4 & 0.05 & 0.9982 & $92(6,4)$ \\
\hline 105 & Pyrazophos & 44.92 & $221.0 \rightarrow 177.0$ & 15 & $221.0 \rightarrow 193.0$ & 10 & 4 & 0.01 & 0.9929 & $94(9,4)$ \\
\hline 106 & Azinphos ethyl & 45.36 & $160.0 \rightarrow 104.0$ & 10 & $160.0 \rightarrow 132.0$ & 5 & 4 & 0.01 & 0.9992 & $91(14,10)$ \\
\hline 107 & Cifluthrin & 49.00 & $163.0 \rightarrow 91.0$ & 12 & $163.0 \rightarrow 127.0$ & 10 & 4 & 0.05 & 0.9836 & $77(4,8)$ \\
\hline 108 & Flucythrinate & 50.00 & $199.1 \rightarrow 107.0$ & 22 & $199.1 \rightarrow 157.0$ & 10 & 4 & 0.05 & 0.9812 & $79(5,7)$ \\
\hline 109 & Deltamethrin & 53.01 & $253.0 \rightarrow 93.0$ & 18 & $253.0 \rightarrow 192.0$ & 30 & 4 & 0.05 & 0.9801 & $83(9,13)$ \\
\hline \multicolumn{11}{|c|}{ Internal standards } \\
\hline IS1 & Aldicarb-D3 (LC) & 9.38 & $211.0 \rightarrow 89.2$ & 19 & $211.0 \rightarrow 119.2$ & 10 & 4 & - & - & - \\
\hline IS2 & Carbofuran-D3 (LC) & 10.34 & $225.0 \rightarrow 123.1$ & 25 & $225.0 \rightarrow 140.5$ & 25 & 4 & - & - & - \\
\hline IS3 & Chlorfenvinphos-D10 (GC) & 17.60 & $263.0 \rightarrow 159.0$ & 15 & $369.0 \rightarrow 101.0$ & 30 & 5 & - & - & - \\
\hline IS4 & Chloropropham (GC) & 11.32 & $213.0 \rightarrow 127.0$ & 15 & $213.0 \rightarrow 171.0$ & 10 & 4 & - & - & - \\
\hline IS5 & Chlorpyrifos-D10 (GC) & 23.60 & $197.0 \rightarrow 169.0$ & 15 & $362.0 \rightarrow 131.0$ & 20 & 5 & - & - & - \\
\hline IS6 & Diazinon-D10 (GC) & 17.81 & $179.1 \rightarrow 137.1$ & 15 & $315.0 \rightarrow 170.0$ & 20 & 5 & - & - & - \\
\hline IS7 & Heptachloro epoxide cis (GC) & 26.30 & $352.8 \rightarrow 262.9$ & 15 & $352.8 \rightarrow 288.9$ & 15 & 4 & - & - & - \\
\hline IS8 & Thiobencarb (LC) & 258.1 & $258.1 \rightarrow 89.1$ & 35 & $258.1 \rightarrow 125.0$ & 19 & 4 & - & - & - \\
\hline
\end{tabular}

a Intra-day.

\section{Results and discussion}

\subsection{Optimization of the instrumental method}

LC-MS/MS and GC-MS/MS provided very low detection limits and could be applied to the identification and confirmation of the peak identities. Two transitions were selected for each analyte included in this study (Table 2). The combination of the transitions and their retention times allowed the pesticide identity to be confirmed.

In this work, we simultaneously investigated 90 pesticides suitable for GC (Table 2) to obtain the most efficient quantitative results with maximum separation. Additionally, we investigated 19 polar pesticides, which were separated by LC (Table 2). Although last generation QqQ analyzers permits the monitoring of co-eluted compounds with a high number of transitions simultaneously in MRM mode [18], and thus, the chromatographic separation is not always a critical stage in the development of a multi-residue method, we assayed several temperature programs (GC method), as well as various gradient programs (LC method), to achieve a good separation of the analytes. The chosen GC and LC operating conditions were those described above (Material and Methods section).

To optimize the triple quadrupole MS/MS conditions, the relevant considerations included the choices of the precursor and product ions and the optimization of the collision energies for the best response of each target compound. For the GC method, we firstly analyzed all the pesticides separately, with the aim of obtaining the full scan spectra and to select the parent ions. After that, another set of analyses was conducted at different collision energy voltages (potential on the second quadrupole) to generate the MS/MS product ions. Similarly, the parent ions described in the bibliography for each one of the 19 LC analytes (usually $\mathrm{M}-\mathrm{H}^{+}$or $\mathrm{M}-\mathrm{H}^{-}$) were confirmed by analyzing the pesticides in separate runs to obtain their full scan spectra. Then, we selected the collision energies and MS/MS product ions after the direct infusion of a solution of each pesticide in methanol $(1 \mu \mathrm{g} / \mathrm{mL})$ 
into the ionization source with the aid of Thermo Fisher Scientific Tune software. The collision energies ( 5 to $39 \mathrm{eV}$ ) are detailed in Table 2.

At the end of this procedure we developed two timed-MRM methods (GC-MS/MS and LC-MS/MS) with two transitions per compound (Table 2). The dwell time was adjusted so that the number of $>$ cycles per second was 10 throughout the chromatographic run to obtain well-shaped chromatographic peaks, low detection limits, and to provide a sufficient number of chromatographic points for all compounds $(>15)$. The peak shapes of all of the analytes in these methods were highly related to the scan time, dwell time, scan rate and number of monitored transitions [19,20]. The final MS/MS conditions used in this study are detailed in Table 2.

The concept of identification points (IPs) for the confirmation stage of mass spectrometry analysis was introduced by the European Commission Decision 2002/657/EC [21]. Meeting the requirements of this regulation, the confirmation of the pesticides included in the present protocol resulted in 4 IPs (two product ions from the same parent ion), or in 5 IPs (two product ions derived from two different parent ions). The resulting number of IPs for each pesticide is also shown in Table 2.

\subsection{Optimization of sample extraction and cleanup}

The liquid-liquid extraction procedure consists of shaking the samples several times in selected organic solvents to extract the pesticide residues from the bulk of the sample matrix. We considered that this method could be convenient for the extraction of pesticides from blood samples. Considering that the pesticides that can be involved in a poisoning episode can belong to different chemical classes, it is critical to select the appropriate solvents to achieve a satisfactory recovery of all of the analytes from the matrix of interest. Organic solvents such as ethanol, methanol, ethyl acetate, hexane and petroleum ether, and their mixtures, including ethanol/ethyl acetate, acetone/hexane, ethyl acetate/acetone/methanol and hexane/dichloromethane have been described for the efficient extraction of pesticides [22]. From the literature we chose various solvent mixtures that would be appropriate for pesticides included in this study, considering their range of polarities. Thus, we assayed the extraction efficacy of mixtures of acetone/hexane (50/50), ethyl acetate/acetone/acetonitrile (40/30/30) and dichloromethane/ethyl acetate/acetone (50/30/20) for all of the pesticides from fortified blood samples. The best combination of purity and recovery was obtained with the dichloromethane/ethyl acetate/acetone (50/30/20) mixture. Therefore we chose this mixture for the extraction.

According to the literature the use of sonication may improve the liquid-liquid extraction efficiency, so we assayed different times of sonication of the samples (5 to $30 \mathrm{~min}$ ). A slight improvement in the recoveries of certain key pesticides (i.e. aldicarb, carbofuran, diazinon and methomyl) was observed with 5 min of sonication, and therefore this step was added to the extraction protocol.

We also included a cleanup step, since the liquid-liquid extraction is a non-selective method and many potentially interfering substances, such as lipids, sugars or pigments, can be co-extracted. Especially lipids should be eliminated to prevent column damage and signal reduction. There are many strategies that can be used for lipid removal: freezing centrifugation, adsorption chromatography, gel permeation chromatography, or sulfuric acid treatment, among others. We chose freezing centrifugation because blood samples yielded relatively clean extracts. Lipids possess a lower melting point than the solvent, and thus with this cleanup method, the lipids can be removed by centrifugation while pesticides remain dissolved in the solvent. According to our experiments, freezing centrifugation was an adequate single-stage cleanup method for blood samples, as it yielded extracts that were suitable for both, LC-MS/MS and GC-MS/MS analyses.

\subsection{Analytical performance}

After the optimization of the analytical methodology we studied the confirmation criteria, precision, linearity, method limits of quantification (LOQs) and repeatability, to evaluate its usefulness for the quantitative determination of pesticides in blood samples.

We only identified the compounds as target analytes if the chromatographic peaks satisfied all of the following criteria: 1) the retention time (tR) of the candidate was the same as that averaged plus or minus three standard deviations $(S D)$ of the $t R(t R \pm 3 S D)$ obtained when six blank samples spiked at the second level of calibration were injected, 2) there was a match with the ion ratios of the standard with a tolerance of $\pm 30 \%$ of absolute ion abundances and 3 ) the $\mathrm{S} / \mathrm{N}$ ratio of the target analyte was $>10$ for a sample extract.

To check whether there were matrix effects we carried out experiments in which blank blood samples were spiked with a mixture of the 109 analytes included in this work at two concentrations: 10.0 and $200.0 \mu \mathrm{g} \mathrm{kg}^{-1}$ (five replicates each). By means of the comparison between the quantifications of the recovered pesticides with those of the same concentrations of pesticides in dissolvent we calculated the recovery percentages (Table 2 ). The results ranged from $68 \%$ to $105 \%$, with most of the recoveries being greater than $85 \%$ at both concentrations. As the most unfavorable RSD was below $15 \%$, we also found that the precision was satisfactory. The inter-day measurement (recoveries and precision during five consecutive days) also yielded an RSD that was below 15\%. Table 2 shows that all results were within the acceptable range and the methods were precise, with RSD values of $3.0-18.0 \%$ for all pesticides.

The quantifications were done against matrix-matched calibration curves, ranging between 0.5 and $500 \mu \mathrm{gg}^{-1}$ (three replicates for each level were analyzed). Calculations were performed using the peak areas. The calibration curves were constructed without including the origin point and were found to have good linearity based on the correlation coefficients $\left(\mathrm{r}^{2}\right)$, which were greater than 0.9801 for all analyses. After performing the residual analysis (values within the range of -10.618 to 11.337 ) we concluded that the linear regression method may be used for quantifications within the range investigated.

\subsection{Application to real samples}

The validated method was applied to the analysis of real samples from two recent cases (May and June of 2013) of pesticide poisoning that were received in the toxicology service of the Institute of Legal Medicine of Las Palmas (Canary Islands, Spain).

\subsubsection{Case 1}

A 79-year-old man was found dead by a friend who went to his house, as the man did not answer the phone. Upon entering he detected a strong odor of "chemical" and found the man on the couch with a belt tied around his neck. According to statements by the sister of the deceased, he lived alone and was being treated for prostate disease and depression. She also reports that he had attempted suicide twice, and for this reason he was being treated in the Mental Health Unit of his area. At autopsy, the remarkable features were: edematous and emphysematous lungs; bloody fluid from the parenchymal cut; presence of yellowish white mucus in the bronchial tubes and trachea; the liver appeared congested; the stomach contained a clear liquid with strong solvent odor, and walls with signs of erosive gastritis; and erosions were also observed in the esophageal mucosa. Gastric content and blood samples were submitted to our laboratory for toxicological analysis. The results of abuse of drugs and alcohol were negative. When we applied the protocol described in this paper to the blood sample in the GC-MS/MS analysis we found the organophosphate insecticide diazinon at a concentration of $6.48 \mu \mathrm{g} / \mathrm{mL}$ (Fig. 1A), which is more than six times higher than the value described by Repetto and Repetto (2007) in a previous fatality [15]. The proposed methodology was also applied to the gastric content sample. We first homogenized the sample and diluted it with ultrapure water (1:10), and we also performed an additional centrifugation in the clean-up step. In Fig. 1B 


\section{A) Blood}
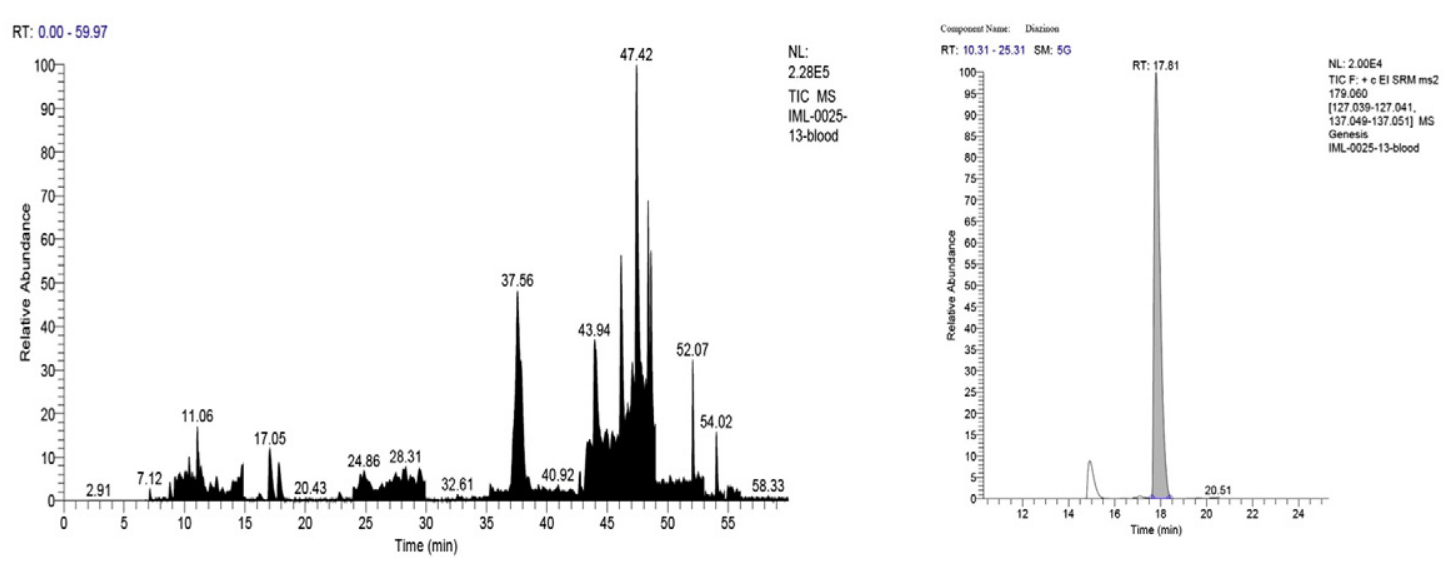

\section{B) Gastric content}
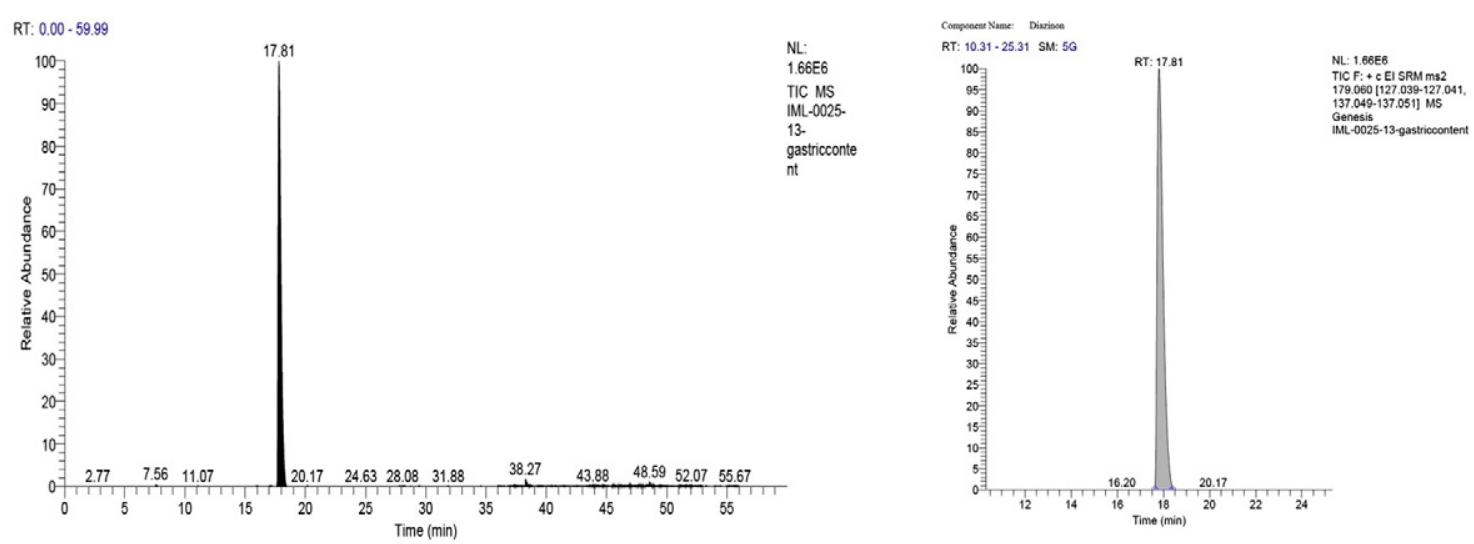

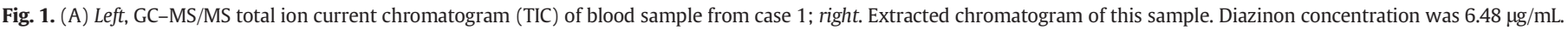
(B) Left, TIC of gastric content sample from case 1. Right, extracted MRM chromatogram of this sample, showing the identification of diazinon.

we show the raw and the filter-extracted chromatograms that we obtained from this sample, in which we clearly identified the diazinon.

\subsubsection{Case 2}

A 63 year old woman was taken to the hospital after eating three tablespoons of vegetable soup that her husband had prepared for her. She did not eat more because "the soup tasted like dirt". According to her own statement, she soon vomited at home and felt strong nausea and abdominal pain. Her son took her to the hospital, and on admission to ICU she displayed marked cholinergic symptoms and was semiconscious. The patient was given pralidoxime and atropine. Her condition gradually improved on days 2 and 3 and she was discharged at $87 \mathrm{~h}$ after admission. A blood sample that was taken on admission was submitted to the laboratory and the described methodology was applied. In LC-ESI-MS/MS analysis the carbamate insecticide aldicarb was detected at a concentration of $2.32 \mu \mathrm{g} / \mathrm{mL}$ (Fig. 2). Days later the police brought to our laboratory a plastic container containing the remains of the soup, which had been located by the son in a dumpster quite out of the marital home. According to the police report, the son suspected of an attempted homicide by her father because of his strange behavior and bad relationship and frequent quarrels they had. By visual examination the soup showed abundant black colored granules (Fig. 3). One gram of this material was diluted in $10 \mathrm{~mL}$ of ultrapure water and subjected to the same method of extraction and chromatographic analysis and the presence of aldicarb was confirmed.

\subsection{Limitations of the methodology}

The proposed methodology has been successfully applied to the identification of pesticides in samples from real human poisoning episodes, allowing their quantification in the case of blood samples. Nevertheless, in spite of being quick, easy and very useful, it should be noted that this methodology has several limitations, such as the use of large amounts of expensive and hazardous organic solvents; the necessity of evaporation of solvents, which is a source of environmental contamination; the multiple steps that suppose a risk of analyte losses; and that very relevant pesticides could not be included (i.e. strychnine, paraquat, alpha-chloralose), since they are chemically very different from the rest, so that additional analysis should be specifically targeted to the identification of these compounds in particular. Besides, the whole procedure is time consuming, and because of the nature of the samples and the extraction and cleanup the methodology cannot be automated and therefore costs remain high.

\section{Conclusions}

We have shown in this paper the applicability of a methodology based on a liquid-liquid extraction followed by a combination of two chromatographic methods (LC and GC) with mass spectrometry detection for the identification and quantification of 109 toxic pesticides in blood samples from human pesticide poisoning episodes. The validation parameters were satisfactory. For all the pesticides we found good 

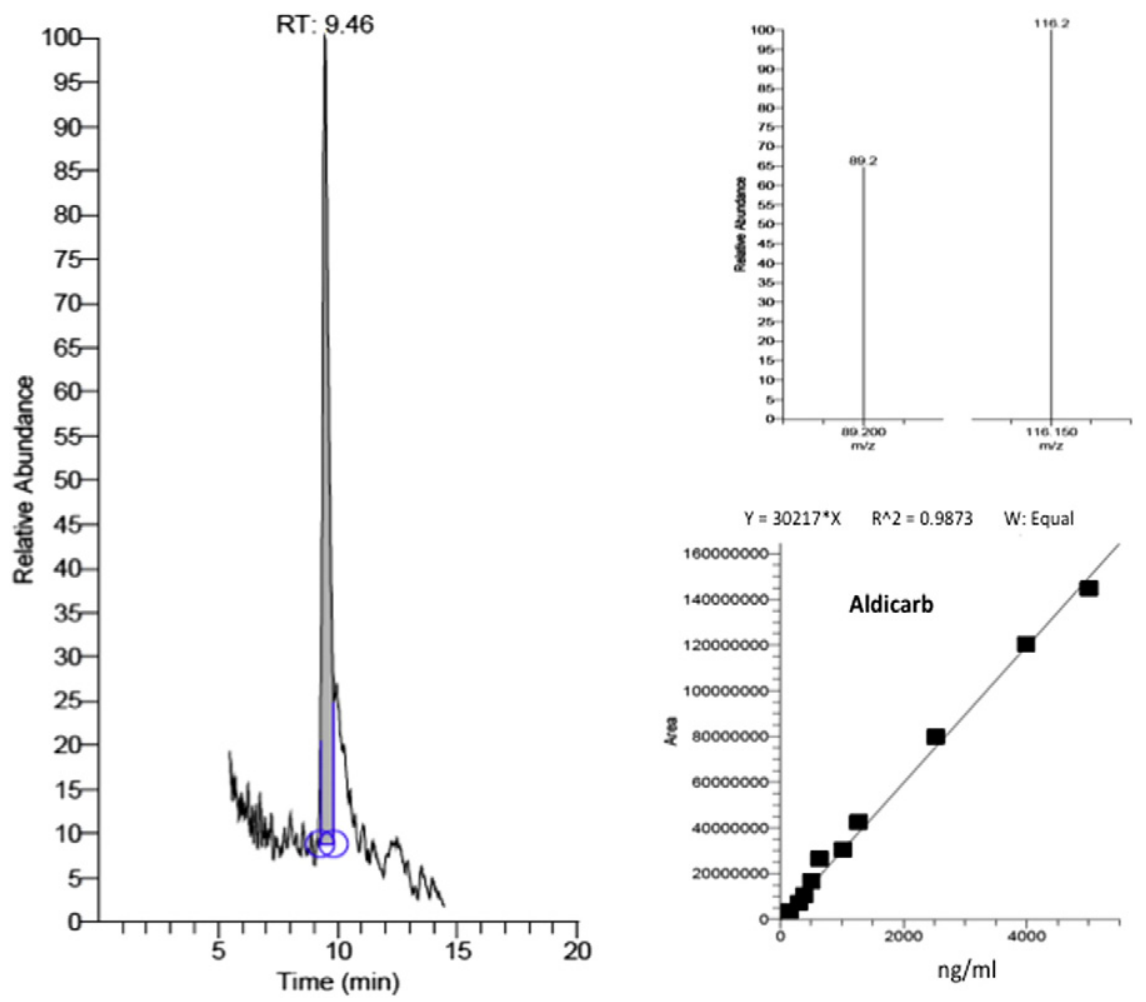

Fig. 2. Left, LC-MS/MS extracted MRM chromatogram of the blood sample from case 2; right, ion ratio, and calibration plot of aldicarb. Concentration was $2.32 \mu \mathrm{g} / \mathrm{mL}$.

linearity (0.5-500 $\mu \mathrm{g} / \mathrm{mL}$, with $\left.r^{2}>0.98\right)$ and low detectability. The recoveries (68 to 105\%) were good, and the precision (RSD $<15 \%$ ) was acceptable. Thus we conclude that this methodology, which is simple, sensitive, and very reliable, may be recommended for its routine application in forensic toxicology laboratories. The applicability of the optimized method was proven in the analysis of samples from two recent poisoning cases. Our results also showed that this methodology is robust enough to be applied to samples different from blood, because the involved pesticide was also identified in gastric contents and food samples.

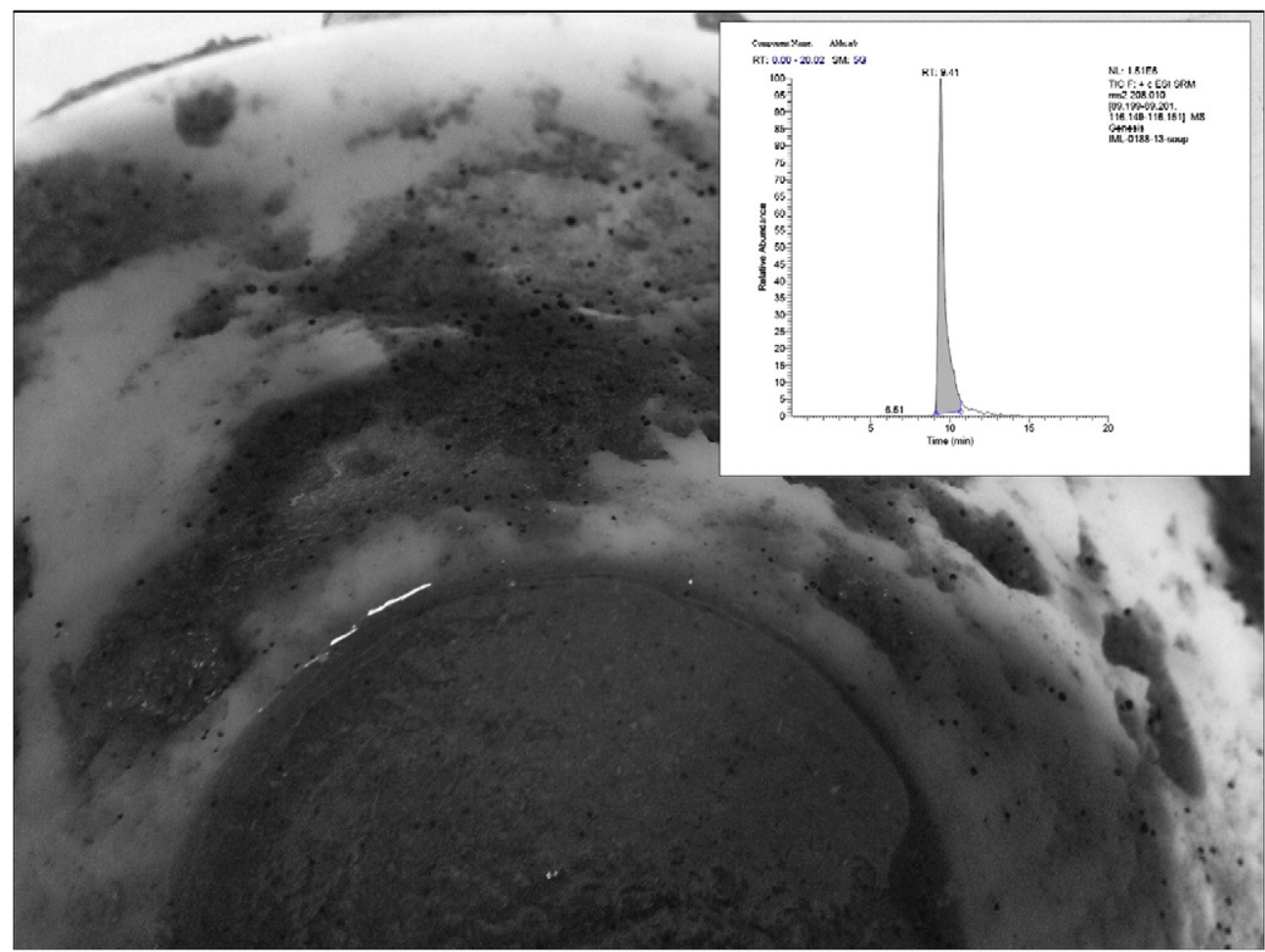

Fig. 3. Vegetable soup showing abundant black colored granules, which were positively identified as aldicarb. 


\section{Conflict of interest}

There are no financial or other relations that could lead to a conflict of interest.

\section{Acknowledgments}

The authors would like to thank to Mrs. María de los Reyes Suárez Hanna and to Dr. Esmeralda Romero-Covo for their technical assistance.

\section{References}

[1] L. Kumar, S.S. Agarwal, K.H. Chavali, S.C. Mestri, Homicide by organophosphorus compound poisoning: a case report, Med. Sci. Law 49 (2009) 136-138.

[2] V.A. Boumba, M. Georgiadis, N. Mirescu, T. Vougiouklakis, Fatal intoxications in a forensic autopsy material from Epirus, Greece, during the period 1998-2010, J. Forensic Sci. 58 (2) (2012) 425-431.

[3] M. Eddleston, M.R. Phillips, Self poisoning with pesticides, BMJ 328 (2004) 42-44

[4] S.P. Singh, A.D. Aggarwal, S.S. Oberoi, K.K. Aggarwal, A.S. Thind, D.S. Bhullar, D.S Walia, P.S. Chahal, Study of poisoning trends in north India-a perspective in relation to world statistics, J. Forensic Legal Med. 20 (2013) 14-18

[5] M. Eddleston, L. Karalliedde, N. Buckley, R. Fernando, G. Hutchinson, G. Isbister, F. Konradsen, D. Murray, J.C. Piola, N. Senanayake, R. Sheriff, S. Singh, S.B. Siwach, L. Smit, Pesticide poisoning in the developing world-a minimum pesticides list, Lancet 360 (2002) 1163-1167.

[6] D.A. Lambropoulou, T.A. Albanis, Methods of sample preparation for determination of pesticide residues in food matrices by chromatography-mass spectrometrybased techniques: a review, Anal. Bioanal. Chem. 389 (2007) 1663-1683.

[7] S. Walorczyk, Development of a multi-residue method for the determination of pesticides in cereals and dry animal feed using gas chromatography-tandem quadrupole mass spectrometry II. Improvement and extension to new analytes, J. Chromatogr. A 1208 (2008) 202-214.

[8] G.F. Pang, C.L. Fan, F. Zhang, Y. Li, Q.Y. Chang, Y.Z. Cao, Y.M. Liu, Z.Y. Li, Q.J. Wang, X.Y $\mathrm{Hu}$, P. Liang, High-throughput GC/MS and HPLC/MS/MS techniques for the multiclass, multiresidue determination of 653 pesticides and chemical pollutants in tea, J. AOAC Int. 94 (2011) 1253-1296.

[9] O.P. Luzardo, N. Ruiz-Suarez, P.F. Valeron, M. Camacho, M. Zumbado, L.A. HenriquezHernandez, L.D. Boada, Methodology for the identification of 117 pesticides commonly involved in the poisoning of wildlife using GC-MS-MS and LC-MS-MS, J. Anal. Toxicol. 38 (3) (2014) 155-163.
[10] K.M. Kasiotis, C. Anagnostopoulos, P. Anastasiadou, K. Machera, Pesticide residues in honeybees, honey and bee pollen by LC-MS/MS screening: reported death incidents in honeybees, Sci. Total Environ. 485-486 (2014) 633-642.

[11] B. Gilbert-Lopez, J.F. Garcia-Reyes, C. Meyer, A. Michels, J. Franzke, A. Molina-Diaz, H. Hayen, Simultaneous testing of multiclass organic contaminants in food and environment by liquid chromatography/dielectric barrier discharge ionizationmass spectrometry, Analyst 137 (2012) 5403-5410.

[12] F. Yang Z. Bian, X. Chen, S. Liu, Y. Liu, G. Tang Analysis of 118 pesticides in tobacco after extraction with the modified QuEChRS method by LC-MS-MS, J. Chromatogr. Sci. 52 (2014) 788-792.

[13] WHO, The WHO Recommended Classification of Pesticides by Hazard and Guidelines to Classification: 2009, Geneva: World Health Organization, 2010, (2009).

[14] SINITOX, Sinitox - sistema nacional de informaçoes toxico farmacológicas, Deaths Reported for Intoxication and Poisoning2010. (Brazil, Available from: http://www. fiocruz.br/sinitox_novo/cgi/cgilua.exe/sys/start.htm?sid=379).

[15] M.R. Repetto, M. Repetto, Tabla de concentraciones de xenobióticos en fluidos biológicos humanos como referencia para el diagnóstico toxicológico (actualización 2007), in: M. Repetto (Ed.), Ampliación de Toxicología de Postgrado, Universidad de Sevilla, Sevilla, 2007.

[16] N. Ruiz-Suarez, L.D. Boada, L.A. Henriquez-Hernandez, F. Gonzalez-Moreo, A. Suarez-Perez, M. Camacho, M. Zumbado, M. Almeida-Gonzalez, M. Del Mar Travieso-Aja, O.P. Luzardo, Continued implication of the banned pesticides carbofuran and aldicarb in the poisoning of domestic and wild animals of the Canary Islands (Spain), Sci. Total Environ. 505 (2015) 1093-1099.

[17] M. Martínez-Haro, R. Mateo, R. Guitart, F. Soler-Rodríguez, M. Pérez-López, A.J. García-Fernández, Relationship of the toxicity of pesticide formulations and their commercial restrictions with the frequency of animal poisonings, Ecotoxicol. Environ. Saf. 69 (2008) 396-402.

[18] C. Liao, P. Yang Z. Xie, Y. Zhao, X. Cheng, Y. Zhang, Z. Ren, Z. Guo, J. Liao, Application of GC-triple quadrupole MS in the quantitative confirmation of polycyclic aromatic hydrocarbons and phthalic acid esters in soil, J. Chromatogr. Sci. 48 (2010) 161-166.

[19] A. Rashid, S. Nawaz, H. Barker, I. Ahmad, M. Ashraf, Development of a simple extraction and clean-up procedure for determination of organochlorine pesticides in soil using gas chromatography-tandem mass spectrometry, J. Chromatogr. A 1217 (2010) 2933-2939.

[20] S. Walorczyk, Development of a multi-residue screening method for the determination of pesticides in cereals and dry animal feed using gas chromatography-triple quadrupole tandem mass spectrometry, J. Chromatogr. A 1165 (2007) 200-212.

[21] 2002/657/EC, Comission Decision Implementing Council Directive $N^{\circ}$ 96/23/CE of August 12, in: Off. J. Eur Union.

[22] J.G. Martins, A. Amaya Chavez, S.M. Waliszewski, A. Colin Cruz, M.M. Garcia Fabila, Extraction and clean-up methods for organochlorine pesticides determination in milk, Chemosphere 92 (2013) 233-246. 